\title{
CAMBIOS PULPARES VASCULARES Y ESTRUCTURALES INDUCIDOS POR FUERZAS ORTODÓNTICAS: UNA REVISIÓN
}

I.udwing J. Delgado C.. "Corlos Alberto Ojeda C.". Humberto Ferreira A., "Lugenio Ordoñez M.

'Odontólogo, U. Santo Tomís, Residente de U año. Fspecialización en Fndodoncia, U. Santo Tomós.

"Odantölogo. U. Nacional. Residente de ll año, Especiolizción en lindoroncia. U. Santo Tomás.

Odontólogo, U. de Cortagena, Residente de Il axio, Especialización en findodoncia, U. Santo Tomás

- Odontólogo, U. Merropolitana de Barranquilla. Residente de II oño. Especialización en Endodoncio. U. Santo Tomás

Autor responsable de correspondencia: Dr. I.udwing Javier Delgado C.

Correo electrónico: Iudwingdel@yahoo.com

\begin{abstract}
RESUMEN
Se ha sugerido que, en ocasiones, las fuerzas ortodónticas producen alteraciones en la angiogénesis, estructura y flujo sanguineo pulpar. Sin embargo, en la literatura se encuentran reportes de estimulación de la angiogénesis a través de los factores de crecimiento: cambios estructurales debidos a fibrosis, apoptosis, degeneraciones pulpares y necrosis; cambios vasculares por una reducción en el flujo songuineo pulpar, y cambios en la confiabilidad de las pruebas de sensibilidad. El propósito de esta revisión es plantear varios cuestionamientos sobre los cambios pulpares vasculares y estructurales inducidos por fuerzas ortodónticas. (Delgado L. Ojeda CA. Ferreira H. Ordoñez F. Cambios pulpares vasculares y estructurales inducidos por fuerzas ortodónticas: Una revisión. Ustasalud Odontologia 2005: 4: 44 - 47 ]
\end{abstract}

Palabras clave: Angiogénesis, movimiento dental, flujo pulpar, vitalidad pulpar.

\section{VASCULAR AND STRUCTURAL PULP CHANGES INDUCED BY ORTHODONTIC FORCES: A REVIEW}

\begin{abstract}
It is thought that. in occasions, the orthodontic forces produce alterations in the angiogenesis, structures and capillary pulpar llow. Nevertheless, it can be found reports of stimulation of the angiogenesis through the growth factors; structural changes due to fibrosis, apoptosis, degenerations pulpares and necrosis; vascular changes by a reduction in the sanguineous tlow to pulpar, and changes in the trustworthiness of the sensitivity tests. The purpose of this article is to raise several questions on the structural and vascular pulpar changes induced by orthodontic forces.
\end{abstract}

Key words: Angiogenesis. Tooth movement, Pulpar flow, Pulpar vitality.

Recibido para publicaciont 23 de noviembre de zont. Aceptado para publicación: 12 de abril de 2005.

\section{INTRODUCCIÓN}

Los movimientos ortodónticos generan cambios estructurales y de respuesta de la pulpa dental. Los procesos de angiogénesis y neovascularización son esenciales en muchos eventos tales como en el desarrollo embriológico. la cicatrización de heridas y los procesos patológicos que involucran el crecimiento de los vasos sanguineos.' Derringer y colaboradores encontraron que la respuesta angiogénica de la pulpa dental a fuerzas ortodónticas era el aumento de los factores de crecimiento angiogénico. ${ }^{2}$

Los efectos del movimiento dental con aparatologia ortodóntica sobre la estructura de la pulpa han sido ampliamente estudiados. Mjör y Stenvik mostraron que las fuerzas intrusivas y extrusivas causaron degeneración de la capa odontoblástica debido a disturbios circulatorios en el tejido pulpar humano. ${ }^{3}$ McDonald y Pittford encontraron que el flujo sanguineo pulpar disminuía (por aproximadamente 32 minutos) cuando eran aplicadas fuerzas ligeras y continuas; esta disminución continua por un periodo extenso de aumento del flujo sanguineo durante 48 horas. ${ }^{4}$

Estos cambios en la estructura pulpar humana producen hipoxia que podría reducir la transmisión nerviosa. Clinicamente, se traduce en una respuesta menos confiable a las pruebas de sensibilidad. ${ }^{3}$ Por 10 tanto, es muy importante realizar cuestionamientos sobre la verdadera acción de las fuerzas ortodónticas en la estructura y vasculatura pulpar.

44 
El propósito de esta revisión es plantear varios cuestionamientos acercad de algunos puntos relacionados con los cambios pulpares vasculares y estructurales inducidos por fuerzas ortodónticas.

\section{¿Los movimientos ortodónticos afectan Ia angiogénesis pulpar?}

Angiogénesis se define como la formación de nuevas estructuras capilares a partir de una vasculatura preexistente.

La formación de vasos sanguíneos es un proceso complejo y de multiples etapas que requiere una serie de eventos celulares en los cuales las células endoteliales. en ciertas áreas, degradan su membrana basal, proliferan y migran dentro del estroma en forma de un brote, el germen capilar; éste se elonga y organiza dentro de las ramificaciones capilares con un lumen. Esos eventos ocurren en respuesta a estímulos angiogénicos. 'Los procesos de angiogénesis y neovascularización son esenciales en muchos eventos como el desarrollo embriológico, la cicatrización, la reparación de heridas (siendo un prerrequisito para un proceso exitoso, incluso en el diente) y los procesos patológicos que involucran crecimiento de vasos sanguíneos como en la formación de tumores.

Durante la dentinogénesis primaria, una rica vasculatura se desarrolla en la zona odontogènica del complejo dentinopulpar, mostrando una fenestración incrementada de vasos para la nutrición de los odontoblastos. Cuando se ha completado la formación de la dentina se observa una disminución de estas fenestraciones y un retiro de los vasos de la capa odontoblástica. En el diente maduro la red capilar es ampliamente confinada a la región subodontoblástica. Los capilares periféricos parecen estar, estrecinamente, relacionados a la actividad de los odontoblastos.

Después de un daño dental, la dentina reparativa o reaccionaria, variantes de la dentinogénesis terciaria, aumenta la reparación de los tejidos e igualmente la revascularización de la pulpa. Este es un evento importante de la respuesta cicatrizal del diente. ${ }^{.9}$

Por lo tanto, se considera que la respuesta angiogénica se refiere a un efecto acumulativo de factores reguladores positivos y negativos. ${ }^{10}$ Un numero de factores de crecimiento polipeptídicos han estado implicados en la iniciación de la respuesta angiogénica, en la regulación de la proliferación endotelial y en la cicatrización de heri- das. ${ }^{11}$ Estos incluyen el factor de crecimiento fibroblástico (FGF2), el factor de crecimiento derivado de las plaquetas (PDGF), el factor de crecimiento epidermal (EGF), el factor de crecimiento transformante (TGF $\beta$ ), y en condiciones de hipoxia e isquemia, factor de crecimiento celular endotelial (VEGF). ${ }^{12}$ Estos factores de crecimiento polipeptídicos pueden estimular la migración, la mitosis y la diferenciación de las células endoteliales en cultivos y pueden inducir la neovascularización en modelos animales.

Es conocido que las fuerzas ortodónticas producen daño mecánico y reacciones inflamatorias en el periodonto: ${ }^{13}$ daño celular, cambios inflamatorios y disturbios circulatorios en la pulpa por la falta de circulación colateral con reducción de los niveles de oxigeno." Durante el curso de un iratamiento prolongado con aparatologia ortodóntica fija, se han observado cambios angiogénicos en respuesta a una repetida estimulación de los factores de crecimiento. ${ }^{15}$

Davidovitch y Rygh concluyeron que no es el tipo de movimiento dental o la fuerza lo que es de importancia. sino la estimulación de los vasos sanguíneos. La activación del sistema vascular es el factor clave. ${ }^{16.17}$ La magnitud de la fuerza no necesariamente debe ser excesiva, incluso pequeñas fuerzas de corta duración de alrededor de cuatro horas pueden ser necesarias para evocar la respuesta celular. ${ }^{19}$

Roberts y colaboradores sugirieron que la matriz de dentina contenía factores de crecimiento angiogénicos responsables de la señalización de la respuesta angiogénica local. que podria aumentarse en los casos en que un evento de reparación se lleve a cabo.'

Derringer y colaboradores encontraron la formación de una red de microvasos que indicaba una respuesta angiogénica en las pulpas de cultivos de este tejido. ${ }^{15}$ Esta respuesta fue significativamente mayor en las pulpas extraidas y estudiadas de los dientes tratados ortodónticamente al compararlas con los dientes del grupo control (sin tratamiento). Sugirieron que existia un incremento en la liberación de los factores de crecimiento asociados con la aplicación de la fuerza ortodóntica. En este estudio, se encontró un incremento del $100 \%$ en el número total de microvasos. ${ }^{15}$

\section{¿Hay cambios estructurales en el tejido pulpar con la aplicación de fuerzas ortodónticas?}

Algunos estudios se han basado en la evidencia de que el tejido pulpar dental responde de manera deletérea a las 
fuerzas ortodónticas, muchas veces, con efecto sobre la vitalidad del diente. ${ }^{19}$ Algunos autores han reportado cambios vasculares en la pulpa dental como respuesta a movimientos ortodónticos. ${ }^{20: 22}$

Nixon y colaboradores, en 1993, encontraron cambios vasculares significativos con un incremento del número de vasos sanguineos pulpares funcionales relacionados con el grupo de aplicación de fuerzas ortodonticas. También, observaron un aumento en la predentina dependiente de la fuerzo hacia el día 5 y 10 del ciclo de movimiento dental. ${ }^{23}$ Guevara y McClugage, en 1980, encontraron que fuerzas intrusivas podrian detener el flujo sanguíneo pulpar en la porción apical llevando a muerte pulpar por un corte de irrigación. ${ }^{22}$

\section{¿Los movimientos ortodónticos afectan el flujo san- guineo pulpar?}

Profitt explicó que la aplicacion de fuerzas ortodónticas podria producir una reacción inflamatoria periodontal pero no pudo demostrar que este efecto fuera más allá de una inflamación moderada de la pulpa..$^{24}$ Aunque las publicaciones histológicas han demostrado que el tejido pulpar reacciona desde un éxtasis vascular circulatorio hasta una necrosis.

Mostafa y colaboradores mostraron que las fuerzas intrusivas y extrusivas causaron degeneración de la capa odontoblástica y disturbios en la circulación del tejido pulpar; reportó la dilatación, la congestión de los vasos sanguíneos y el edema del tejido pulpar. ${ }^{25}$

Turley y colaboradores aplicaron fuerzas ortodónticas extrusivas a dientes de perros intruidos, observaron que la mitad de los dientes estaban totalmente necróticos, los demás presentaban degeneración y calcificación del tejido pulpar. ${ }^{26}$

Moss y Timms notaron marcados cambios pulpares incluyendo depósitos de dentina secundaria y presencia de pólipos pulpares. ${ }^{27}$ Hamilton y Gutmann reportaron que los movimientos ortodónticos pueden causar una respuesta inflamatoria y/o degenerativa de la pulpa en dientes con una completa formación apical. El impacto sobre la pulpa se observa principalmente sobre el sistema neurovascular, el cual relaciona los neurotransmisores especificos (neuropéptidos) que pueden influenciar tanto en el flujo sanguíneo como en el metabolismo celular. ${ }^{2 x}$
Oppenheim mostró signos de degeneración pulpar severa. Estos hallazgos se enfocaron a la falta de circulación colateral de la pulpa durante el movimiento dental, por esto es recomendable el uso de fuerzas ligeras e intermitentes para reducir el daño al tejido dental y de esta manera proporcionar mayor tiempo de reparación. ${ }^{29}$

Labart y colaboradores reportaron un aumento en la "respiración pulpar" como resultado de las fuerzas ortodónticas que habian sido aplicadas de manera continua sobre incisivos de ratones en erupción. Se observó que el tejido alrededor del hueso y del ligamento periodontal eran semejantes al de un diente con ápice abierto y foramen grande."

Seltzer y Bender reportaron alteraciones en la vascu latura pulpar con una subsiguiente alteración del metabolismo de las células pulpares causando un aumento en el deposito de dentina reparativa tanto en la porción coronal como en la radicular. ${ }^{31}$

Bunner y Jonson concluyeron que las alteraciones de los axones intrapulpares eran mínimas y que no progresaban con movimientos ortodónticos conservadores. ${ }^{32}$

\section{CONCLUSIÓN}

Los estudios acerca del efecto del movimiento ortodóntico sobre la pulpa dental han demostrado una inducción de factores de crecimiento angiogénico. lo que favorece la angiogénesis pulpar, el aumento en el número de vasos sanguíneos, el aumento de generación de predentina, el desplazamiento de los odontoblastos, la reducción inicial del flujo sanguineo pulpar con el subsiguiente aumento para terminar finalmente con la normalización del flujo. Los movimientos ortodóncicos leves producen efectos reversibles en el tejido pulpar humano.

\section{BIBIJOGRAFIA}

1. Roberts-Clark DJ. Smith AJ. Angiogenic growh factors in human dentin matrix. Arch Oral Biol 2000; 45: 1013 - 1016.

2. Derringer KA, Jaggers D], Linden RWA. Angiogenesis in human dental pulp following orthodontit touth movement. J Dent Res 1996 75: $1761-1766$

3. Stenvik A. Mjör IA. Pulp and dentin reaction to experimental tooth intrusion. A histulogic study of the initial changes. Am J Orthod 1970 57: $370-385$.

46 
4. McDonald F. Pittford TR. Blood flow changes in permanent maxillary canines during retractions. Fur J Orthod 1994; 16: 1 - 9.

5. Rowe AHR, Pituford TR. The assessment of puipal vitality. Int Findod J 1990: 23: $77-83$.

6. Baume LJ. 1980. The biology of the pulp and denune. En: Myers HM. Monographs in oral science. Basel: Karger; 1980.

7. Yoshida S, Ohshima H. Distribution and organization of peripheral capillaries in dental pulp and their relationship to odontoblasts. Anat Rec 1996: 245: 313 - 326.

8. Lesot H. Begue-Kim C, Kubler MIJ, Meyer JM. Smith AJ, Cassidy N, Ruch JV. Experimental induction of odontoblast differentiation. Cell Mater 1993: 3: 201 - 217.

9. Jin $H$. Thomas HF, Chen J. Wound healing and revascularization: a histologic observation of experimental tooth root fracture. Oral Surg Oral Med Oral Parh Oral Kad Endod 1996; 81: 26 - 30.

10. Folkman J, Klagsbrun M. A family of angiogenic peptides. Nature 1987: 329: $671-672$.

11. Folknan J. Klagsbrun M. Angiogenic fnctors. Science 1987; 235: 442 457 .

12. Harik SI, Hritz MA, LaManna JC. Hypoxia induced brain angiogenesis in the adult rat. J Physial 1995; 485: 525 - 530.

13. Rygh I, Bowling K, Hovlandsdal I., Williams S. Activation of the vascular system: a main mediator of periodontal fiber remodeling in orthodontic tooth movement. Am J Orthod 1986; 89: 453 - 468.

14. Mostaza YA. Iskander K, el Mangoury NH. Iatrogenic pulpal reactions 10 orthodontic extrusion. Am J Ortho Dentofac Orthop 1991: 99: 30 - 34.

15. Derringer KA, Jaggers DJ, Linden RWA. Angiogenesis in human dental pulp following orthodontic tooth movement. J Dent Res 1996; 75: $1761 \cdot 1766$.

16. Davidovitch Z. Cell biology assaciated with orthodontic tooth movement. Fn: Berkovitz BKB. Moxham B]. Newman HN. The periodontal in health and disease. 2nd ed. Orlando: Mosby Yearbook: 1995. p.259 - 277.

17. Rygh P. Bowling K, Hovlansdal L., Williams S. Activation of the vascular system: a main mediator of periodantal remodeling in orthodontic tooth movement. Am J Orhod 1986; 89: 453468.

18. Roberts WE, Ferguson D. Cell Kinetics of the periodontal ligament. En: Norton and Burstone, editors. The biology of tooth movement. London: CRC Press; 1989. p. 55 - 71.

19. Hamersky P. Weimar $A$, Taintor J. The effect of orthodontic force application on the pulpal tissue respiration rate in human premolar. Am J Orthod 1980: 77: $368-378$.

20. Aisenberg MS. The tissues und changes involved in orthodontic tooth movements. Am . Orthod 1948: 34: $854 \quad 859$.
21. Anstendig HS, Kronman JH. A histological study of pulpal reaction to orthodontic tooth movement in dogs. Angle Orthod 1972: 42: 50 - 55.

22. Guevara MH, McClugage SG. Elfects of intrusive forces upon the microvasculature of the dental pulp. Angle Orthod 1980; 50: $129-134$.

23. Nixon CE, Saviano JA, King GJ, Keeling SD. Histomorphometric study of dental pulp during orthodontic tooth movement. J Endod 1993; 17: $13-16$.

24. Profitt WR, lields HW. Contemporary orthodontics. St Louis: Mosby -year Book, 1993: 266 - 281.

25. Mostafa YA, Iskander KG, El-Mangoury NH. Iatrogenic pulpar reactions to orthodontic extrusion. Am J Orthod Dentofac Orthop 1991: $99: 30-34$.

26. Turiey PK, Joiner MW, Helistrom S. The eflect of orthodontic extrusion on traumatically intruded teeth. Am J Orthod 1984: 85: 47 - 56.

27. Moss JP. Timms DJ. A histological investigation inio the effects of rapid maxillary expansion on the teeth and their supporting tissues. Trans Eur Orthod Soc 1971: $263 \cdot 271$.

28. Hamilton RS, Gutmann JL, Endodontic - urthodontic relationships: a review of integrated treatment planning challenges. Int Endod J 1999; $32: 343 \cdot 360$.

29. Oppenheim $A$. Biologic onhodontic therapy and reality. Angle Orthod 1936; 6: 5 - 38

30. 1, abart WA, Thintor JE, Diyer JK, Weimer AD. The effect of orthodontic forces on pulp respiration in the rat incisor. J Endod 1980; 6: 724 - 727.

31. Seltzer S. Bender IB. The dental pulp. 3rd edition. Philadelphia: J.B. I.ippincot Company. 1984: $210-211$.

32. Bunner M, Jahnson D. Quantitative assessment intrapulpar axan response to orthodontic movement. Am J Orthod 1982: 77: 368 - 378. 